\title{
NON-STANDARD 3-SPHERES LOCALLY FOLIATED BY ELASTIC HELICES
}

\author{
JOSÉ L. CABRERIZO and MANUEL FERNÁNDEZ \\ Depto. de Geometría y Topología, Facultad de Matemáticas, Universidad de Sevilla, Apdo. Correos 1160, \\ 41080-Sevilla, Spain \\ e-mail: jaraiz@cica.es andmafernan@cica.es
}

(Received 20 October, 1999)

\begin{abstract}
In this note we use the Hopf map to construct a family of metrics in the 3-sphere parametrized on the space of positive smooth functions in the 2-sphere. All these metrics make the Hopf map a Riemannian submersion. Also, the fibres are all geodesics if and only if the metric comes from a constant function and so, we have a Berger 3-sphere. Every geodesic in a 3-dimensional Riemannian manifold is a minimum for each elastic energy functional. Therefore, we characterize those functions on the 2-sphere that locally give metrics which have all the fibres being elastica, i.e., critical points of those functionals. Some applications are given including one to the Willmore-Chen variational problem.
\end{abstract}

1991 Mathematics Subject Classification. 53C15, 53C40.

1. Introduction. The Willmore-Chen functional [5] is defined on the space of inmersions, $I(N, P)$, of an $n$-dimensional compact smooth manifold $N$ into a semiRiemannian manifold $(P, \bar{g})$ by

$$
\mathcal{W}(\varphi)=\int_{N}\left(\bar{g}(H, H)-\tau_{e}\right)^{n / 2} d v
$$

where $H$ and $\tau_{e}$ denote the mean curvature vector field and the extrinsic scalar curvature function of $\varphi$, respectively, and $d v$ is the volume element of $\varphi^{*}(\bar{g})$ on $N$.

Since the group of conformal transformations of $(P, \bar{g})$ preserves this functional [4], it is also called the conformal total tension functional, an it states a variational problem in $(P,[\bar{g}])$, where $[\bar{g}]$ is the conformal structure defined by $\bar{g}$. The critical points of $\mathcal{W}$ are known as Willmore-Chen submanifolds. Certainly, this is the natural extension to highest dimensions of the Willmore functional which corresponds with $n=2$, and now its critical points are the Willmore surfaces [10].

The reduction of symmetry method gives a strong relationship between this variational problem and another one associated with a certain elastic energy functional. For example, let $P$ be a principal fibre $G$-bundle $(G$ being an $r$-dimensional compact Lie group) endowed with a principal flat connection over a semi-Riemannian manifold $(M, g)$. If $\bar{g}$ is a metric on $P$ obtained by the Kaluza-Klein mechanism, then the principle of symmetric criticality [9] can be used to produce symmetric solutions to the Willmore-Chen variational problem in $(P,[\bar{g}])$. These solutions are associated with the critical points of the elastic energy functional

$$
\mathcal{F}^{r}(\gamma)=\int_{\gamma} \kappa^{r+1} d s,
$$


defined on the space of closed curves $\gamma$ in $(M, g)$, where $\kappa$ denotes the corresponding curvature function [2]. We call $r$-elasticae to the critical points of $\mathcal{F}^{r}$, and again observe that this notion naturally extends the classical one of free elastic curves, which is obtained for $r=1$ [7]. Every closed geodesic in $(M, g)$ is automatically an $r$ elastica.

On the other hand, if $\left(\mathbb{S}^{2}, g\right)$ is the standard round 2-sphere with radius $1 / 2$, the usual Hopf map $\pi: \mathbb{S}^{3} \longrightarrow \mathbb{S}^{2}$ is a principal fibre $\mathbb{S}^{1}$-bundle which admits a canonical principal connection $\omega$ with non-trivial holonomy. For every positive smooth function $f$ on $\mathbb{S}^{2}$, we construct on $\mathbb{S}^{3}$ the metric $\bar{g}^{f}=\pi^{*}(g)+(f \cdot \pi)^{2} \omega^{*}\left(d t^{2}\right)$. It is not difficult to see that all the fibres in $\left(\mathbb{S}^{3}, \bar{g}^{f}\right)$ are geodesics if and only if $f=a$ is a constant and so, $\bar{g}^{a}$ is a Berger metric, i.e. $\left(\mathbb{S}^{3}, \bar{g}^{a}\right)$ is up to a constant factor, isometric to a distance sphere in $\mathbb{C} P^{2}$ or its dual.

In this note, we study the following natural problem.

Given an open subset $U$ in $\mathbb{S}^{2}$, characterize those funtions $f$ such that all the fibres in $\pi^{-1}(U)$ are r-elastica in $\left(\mathbb{S}^{3}, \bar{g}^{f}\right)$.

We also obtain some applications including one that shows the existence of nontrivial conformal structures which are foliated by equivariant Willmore-Chen submanifolds.

2. Some preliminaires. Let $\pi: \mathbb{S}^{3} \longrightarrow \mathbb{S}^{2}$ be the usual Hopf fibration. Here $\mathbb{S}^{3}$ is viewed as the unit 3 -sphere in $\mathbb{C}^{2}$ so that $\bar{g}$ will denote its standard metric of constant curvature 1 . We define a global vector field $V$ on $\mathbb{S}^{3}$ by: $V(z)=i z$ for any $z \in \mathbb{S}^{3}$. We use $V$ and $\bar{g}$ to define the canonical principal connection $\omega$ in this principal fibre $\mathbb{S}^{1}$ bundle. In particular, if we choose on the base $\mathbb{S}^{2}$ the metric $g$ of constant Gaussian curvature 4 , then $\pi:\left(\mathbb{S}^{3}, \bar{g}\right) \longrightarrow\left(\mathbb{S}^{2}, g\right)$ is a Riemannian submersion with geodesic fibres. The following O'Neill formulae are well known. (See [8].)

$$
\begin{aligned}
\bar{\nabla}_{\bar{X}} \bar{Y} & =\overline{\nabla_{X} Y}-\bar{g}(i \bar{X}, \bar{Y}) V, \\
\bar{\nabla}_{\bar{X}} V & =\bar{\nabla}_{V} \bar{X}=i \bar{X} \\
\bar{\nabla}_{V} V & =0
\end{aligned}
$$

where $\bar{\nabla}$ and $\nabla$ stand for the Levi-Civita connection of $\bar{g}$ and $g$, respectively, and overbars means horizontal liftings.

For any positive smooth function $f$ on $\mathbb{S}^{2}$ and $\varepsilon= \pm 1$, we define the semiRiemannian generalized Kaluza-Klein metric $\bar{g}^{f}$ on $\mathbb{S}^{3}$ by

$$
\bar{g}^{f}=\pi^{*}(g)+\varepsilon(f \cdot \pi)^{2} \omega^{*}\left(d t^{2}\right),
$$

where $d t^{2}$ is the standard metric on $\mathbb{S}^{1}$. Then $\pi:\left(\mathbb{S}^{3}, \bar{g}^{f}\right) \longrightarrow\left(\mathbb{S}^{2}, g\right)$ is still a semiRiemannian submersion. Notice that $\bar{g}^{f}$ is Riemannian or Lorentzian according to $\varepsilon$ is +1 or -1 , respectively. Although in this note we will work in the Riemannian case, similar conclusions can be obtained in the Lorentzian one. For the sake of simplicity, we shall write $f$ instead of $f \cdot \pi$. Let $T=\frac{1}{f} V$ be the $\bar{g}^{f}$-unit tangent vector field to the fibres. Then, a standard computation involving some well-known facts from the theory of semi-Riemannian submersions allows us to obtain the corresponding O'Neill formulae: 


$$
\begin{aligned}
\bar{\nabla}_{\bar{X}}^{f} \bar{Y} & =\overline{\nabla_{X} Y}-\bar{g}^{f}(i \bar{X}, \bar{Y}) V, \\
{[T, \bar{X}] } & =\frac{\bar{X}(f)}{f} T, \\
\bar{\nabla}_{T}^{f} T & =-\operatorname{grad}(\log f),
\end{aligned}
$$

where $\bar{\nabla}^{f}$ and grad stand for the Levi-Civita connection and the gradient of $\bar{g}^{f}$, respectively.

3. The fibres in a generalized Kaluza-Klein metric. Recall that a helix in a semiRiemannian manifold is a curve which has constant all its curvature functions. Notice also that the fibres are geodesics, and so helices, in a generalized KaluzaKlein metric on $\mathbb{S}^{3}$ if and only if $f$ is a constant (see equation (2.7)). More generally, if $p \in \mathbb{S}^{2}$, then $\pi^{-1}(p)$ is a geodesic in $\bar{g}^{f}$ if and only if $p$ is a critical point of $f$. Otherwise, let $\kappa$ and $N$ be the curvature and the unit principal normal of $\pi^{-1}(p)$ in $\bar{g}^{f}$, respectively. Then, we combine equation (2.7) with the first Frenet equation of the fibre to obtain

$$
-\operatorname{grad}(f)=f \kappa N
$$

In particular we observe that the fibres have constant curvature $\kappa=\frac{|\operatorname{grad}(f)|}{f}$.

Let $\tau$ and $B$ be the torsion and the unit binormal of a fibre in $\bar{g}^{f}$. Then we combine the formula (2.6) with the second Frenet equation to have

$$
\bar{\nabla}_{N}^{f} T=-\tau B .
$$

Let $\Sigma$ be the set of critical points of $f$. It is not difficult to see that $\{T, N\}$ span an involutive distribution on $\mathbb{S}^{3}-\pi^{-1}(\Sigma)$. Furthermore, every leaf of this foliation can be regarded as a Hopf tube shaped on a curve on $\mathbb{S}^{2}$, i.e., the leaves are as $\pi^{-1}(\gamma)$, where $\gamma$ is an inmersed curve in $\mathbb{S}^{2}$. Notice that these tubes, $S_{\gamma}$, can be parametrized by $\Phi: I \times \mathbb{R} \longrightarrow \mathbb{S}^{3}$ as follows:

$$
\Phi(s, t)=e^{i t} \bar{\gamma}(s)
$$

where $I$ is the domain of $\gamma$ and $\bar{\gamma}$ denotes a horizontal lift of $\gamma$. It should be observed that in this parametrization, the coordinate curves $t=$ constant generate the $N$-flow while those curves obtained for $s=$ constant are fibres. The unit normal vector field to $S_{\gamma}$ in $\left(\mathbb{S}^{3}, \bar{g}^{f}\right)$ coincides with the unit binormal to the fibre. Now, one can compute [1] the shape operator, $A^{f}$, of $S_{\gamma}$ in $\left(\mathbb{S}^{3}, \bar{g}^{f}\right)$. In the orthonormal basis $\left\{T=\frac{1}{f} \Phi_{t}\right.$, $\left.N=\Phi_{s}\right\}$, it is given by the matrix:

$$
A^{f}=\left(\begin{array}{cc}
-B(\log (f)) & f \\
f & \rho
\end{array}\right),
$$

where $\rho$ stand for the curvature of $\gamma$ in $\left(\mathbb{S}^{2}, g\right)$.

On the other hand, formula (3.2) shows that $\bar{\nabla}_{N}^{f} T$ is normal to $S_{\gamma}$ and so

$$
\bar{\nabla}_{N}^{f} T=\bar{g}^{f}\left(A^{f}(N), T\right) B=f B .
$$


Now, we compare this formula with (3.2) to deduce the following result.

Proposition 1. For any positive smooth function $f$ on $\mathbb{S}^{2}$, the fibres of $\pi:\left(\mathbb{S}^{3}, \bar{g}^{f}\right) \longrightarrow\left(\mathbb{S}^{2}, g\right)$ are helices in $\left(\mathbb{S}^{3}, \bar{g}^{f}\right)$ with curvature $\kappa$ and torsion $\tau$ given by

$$
\kappa=\frac{|\operatorname{grad}(f)|}{f} \text { and } \tau=-f
$$

REMARK 1. Notice that the fibres of $\pi$ in Proposition 1 are trivially helices because the $\mathbb{S}^{1}$-action on $\mathbb{S}^{3}$ is carried out throughout isometries of $\left(\mathbb{S}^{3}, \bar{g}^{f}\right)$. However, we shall need these particular values of $\kappa$ and $\tau$ in the next section.

4. Elasticity of fibres. Let $\Omega$ be the manifold of regular closed curves in a semiRiemannian manifold $\left(M, d \sigma^{2}\right)$. For any natural number $r$, define an elastic energy functional $\mathcal{F}^{r}: \Omega \longrightarrow \mathbb{R}$ by

$$
\mathcal{F}^{r}(\gamma)=\int_{\gamma}\left(\kappa^{2}\right)^{\frac{r+1}{2}} d s
$$

where $\kappa$ denotes the curvature function of $\gamma \in \Omega$, and we write the integrand in this form to point out that it is an even function of the curvature. The variational problems associated with these functionals were considered in [2], [3]. The critical points of $\mathcal{F}^{r}$ are called r-elasticae (or $r$-elastic curves), and the Euler-Lagrange equations characterizing these curves were computed there.

In particular, since the fibres of $\pi:\left(\mathbb{S}^{3}, \bar{g}^{f}\right) \longrightarrow\left(\mathbb{S}^{2}, g\right)$ are helices, we use those equations to deduce that a fibre is an $r$-elastica if and only if

$$
\kappa^{r}\left((r+1) \bar{R}^{f}(N, T) T+\left(r \kappa^{2}-(r+1) \tau^{2}\right) N\right)=0,
$$

where $\bar{R}^{f}$ denotes the curvature operator associated with $\bar{g}^{f}$.

As a consequence of this formula, we see that every geodesic fibre is automatically an $r$-elastica for any natural number $r$. In other words, for any $p \in \Sigma$, the fibre $\pi^{-1}(p)$ is an $r$-elastica in $\left(\mathbb{S}^{3}, \bar{g}^{f}\right)$ for arbitrary $r$.

Let $U$ be an open subset of $\mathbb{S}^{2}-\Sigma$. The problem is to characterize those positive smooth functions $f$ on $U$ in order for $\pi^{-1}(p)$ to be an $r$-elastica in $\left(\mathbb{S}^{3}, \bar{g}^{-f}\right)$ for any $p \in U$. To solve this problem, we only need to compute the curvature term appearing in equation (4.1). A straightforward calculus involving some formulae obtained in the last section gives

$$
\bar{R}^{f}(N, T) T=\left(N(\kappa)+\tau^{2}+\frac{N(f)}{f} \kappa\right) N+\kappa \bar{\nabla}_{N}^{f} N
$$

and so it can be combined with equation (4.1) and Proposition 1 to deduce the following.

Proposition 2. Let $U$ be an open subset of $\mathbb{S}^{2}-\Sigma$. Then all the fibres in $\pi^{-1}(U)$ are r-elastica in $\left(\mathbb{S}^{3}, \bar{g}^{-}\right)$if and only if 
(1) the unitary field given by $N=-\frac{\operatorname{grad}(f)}{|\operatorname{grad}(f)|}$ defines a unit speed geodesic flow on
$\pi^{-1}(U)$,

(2) along this $N$-flow, f evolves according to

$$
(r+1) f N(N(f))-r(N(f))^{2}=0 .
$$

Corollary 1. Let $p$ a point of $\mathbb{S}^{2}$ and denote by $-p$ its antipode. We define $U=\mathbb{S}^{2}-\{p,-p\}$ and $f: U \longrightarrow \mathbb{R}$ by $f(x)=(d(x, p))^{r+1}$, where $d(x, p)$ denotes the distance in $\mathbb{S}^{2}$ from $x$ to $p$. Then, $\left(\pi^{-1}(U), \bar{g}^{f}\right)$ admits a foliation with leaves being r-elastica. Furthermore, this is a subfoliation of a foliation in $\left(\pi^{-1}(U), \bar{g}^{f}\right)$ with leaves being flat tori with constant mean curvature.

In the next result, we choose $(U, f)$ as in Corollary 1 .

COROllary 2. Let $G$ be a compact Lie group of dimension $r$ endowed with a biinvariant metric d $\sigma^{2}$. Let $H$ be a closed subgroup of the fundamental group $\pi_{1}\left(\pi^{-1}(U)\right)$ and $\phi: \pi_{1}\left(\pi^{-1}(U)\right) / H \longrightarrow G$ a monomorphism.

(1) There exists a principal fibre G-bundle, $\eta: P \longrightarrow \pi^{-1}(U)$ which admits a principal flat connection $\theta$.

(2) The metric $h=\eta^{*}\left(\bar{g}^{f}+\theta^{*}\left(d \sigma^{2}\right)\right)$ on P defines a conformal structure, [h], on $P$ which is foliated by $(r+1)$-dimensional G-invariant, Willmore-Chen submanifolds which have constant mean curvature in the metric $h$.

Proof. The way to construct $(P, \theta)$ is well-known [6]. To show the second statement, we first notice that the space of $(r+1)$-dimensional compact $G$-invariant submanifolds of $P$ can be identified with $Q=\left\{\eta^{-1}(\alpha) \mid \alpha\right.$ is a closed immersed curve in $\left.\pi^{-1}(U)\right\}$. The Willmore-Chen functional $\mathcal{W}: \bar{Q} \longrightarrow \mathbb{R}$ is defined on the space $\bar{Q}$ of $(r+1)$-dimensional compact submanifolds of $P$ and it only depends on the conformal structure. Since the natural action of $G$ on $P$ is carried out throughout isometries of $(P, h)$, it preserves $\mathcal{W}$ and hence, we can apply the principle of symmetric criticality (see [9]). Therefore, to obtain $G$-invariant Willmore-Chen submanifolds in $(P,[h])$ we only need to compute critical points of $\mathcal{W}$ but restricted to $Q$. However, this restriction can be computed to obtain that $\mathcal{W}\left(\eta^{-1}(\alpha)\right)$ is a constant multiple of $\mathcal{F}^{r}(\alpha)$ (see [2]). Consequently, $\eta^{-1}(\alpha)$ is Willmore-Chen in $(P,[h])$ if and only if $\alpha$ is an $r$-elastica in $\left(\pi^{-1}(U), \bar{g}^{f}\right)$. Now the second statement follows from Corollary 1.

ACKnOWLedgements. The authors would like sincerely to thank M. Barros for his valuable comments and suggestions.

\section{REFERENCES}

1. M. Barros, A. Ferrández and P. Lucas, Lawson's type problems in non-standard 3-spheres, Quart. J. Math. Oxford Ser. (2) 50 (1999), 385-388.

2. M. Barros, A. Ferrández P. Lucas and M. A. Meroño, A criterion for reduction of variables in the Willmore-Chen variational problem and its applications, Trans. AMS, 352 (2000), 3015-3027.

3. M. Barros, A. Ferrández P. Lucas and M. A. Meroño, Willmore tori and WillmoreChen submanifolds in pseudo-Riemannian spaces, J. Geom. Physics 28 (1998), 45-66. 
4. B. Y. Chen, Some conformal invariants of submanifolds and its applications, Boll. Un. Mat. Ital. 10 (1974), 380-385.

5. B. Y. Chen, Total mean curvature and submanifolds of finite type (World Scientific Pub. Co., 1984).

6. S. Kobayashi and K. Nomizu, Foundations of differential geometry (John Wiley, 1963(I), 1969(II)).

7. J. Langer and D. A. Singer, The total squared curvature of closed curves, J. Differential Geom. 20 (1984), 1-22.

8. B. O’Neill, Semi-Riemannian geometry (Academic Press, 1983).

9. R. S. Palais, Critical point theory and the minimax principle, Global Analysis, Proc. Sympos. Pure Math. 15 (1970), 185-212.

10. T. J. Willmore, Note on embedded surface, An. Sti. Univ. Al. I. Cuza Iasi Sect. Ia Mat. 11B (1965), 493-496. 